\title{
Using an analytical model of simulation in the design of light-weight armours
}

\author{
Ramón Zaera \\ Dpto. de Ingeniería. Escuela Politécnica Superior \\ Universidad Carlos III de Madrid. Madrid, Spain. \\ Vicente Sánchez-Gálvez \\ Dpto. Ciencia de Materiales. E. T. S. Ingenieros de Caminos, Canales y Puertos \\ Universidad Politécnica de Madrid. Madrid, Spain.
}

This paper presents a model for the simulation of the impact of projectiles of high kinetic energy on light-weight ceramic/metal armours. The work was financed jointly by the Defence Ministries of Spain, Italy, Holland and Denmark as part of a project between nations for the development of tools for the design of this type of armour. The model consists of a series of differential equations incorporating the most essential phenomena of the impact process formulated after a close study of a series of real fire tests. A nucleus of calculation into which these equations may be integrated in time, and also of a user interface, are features of a practical preliminary design tool whose efficacy is amply confirmed by test results.

Keywords: projectile, ceramic armour, ballistic impact.

\section{Introduction}

Weight is a key factor in the design of materials for protection against impact for moving objects -vehicles, civil or military aircraft, and personnel of security and defense corps-. Hence the interest in developing light-weight armour, which made great headway with the introduction of ceramic/metal armour at the end of the sixties [1], 60\% lighter than the steel armours then in use.

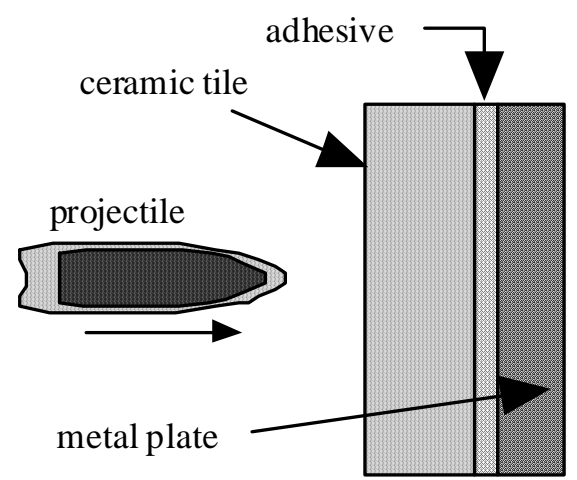

Figure 1. Layout of the ceramic/metal armour.

This type of protection is composed of a tile of ceramic material that receives the impact, and a metallic backplate. The two components are normally joined by a thin layer of adhesive (Fig. 
1). The very hard ceramic material serves to erode the head of the projectile while the metal plate withstands the fragments of the ceramic and absorbs the kinetic energy of the projectile in its penetration. The protection can be used alone, to arrest the projectile, or it can serve as an additional protection over a backing armour.

A simulation model has been developed by the authors for the design of armour with the above characteristics for protection against small and medium caliber projectiles.

\section{Design methods for ceramic/metal protections.}

The traditional method of design was an empirical one of carrying out a large number of firing tests. Not only was it costly but it had the added disadvantage that the results cannot be extrapolated to configurations other than those tested. An alternative method is by simulation by hydrocodes that integrate the differential equations of the mechanics of continuous media by means of finite differences or of finite elements. This is an expensive technique on account of the high market price of the programmes; the simulation of an impact is a long process, at least 5-10 hours in a personal computer, and to be able to use and interpret the programmes the operator needs a high degree of competence.

So a design engineer needs a low-cost tool that would enable him to solve a problem of impact in the shortest possible time, that would be easy to use, and that would give the required precision. The answer to this is found in the analytical simulation models that present the impact process by straightforward equations, and parameters that can be assigned by simple experiment. Since they are quick and easy to use, they allow a simulation of a large number of impact problems in the early stages of design, limiting the number of materials to be considered and the thicknesses to be covered. This means a great saving of the time and money that would be spent if the earlier methods were used.

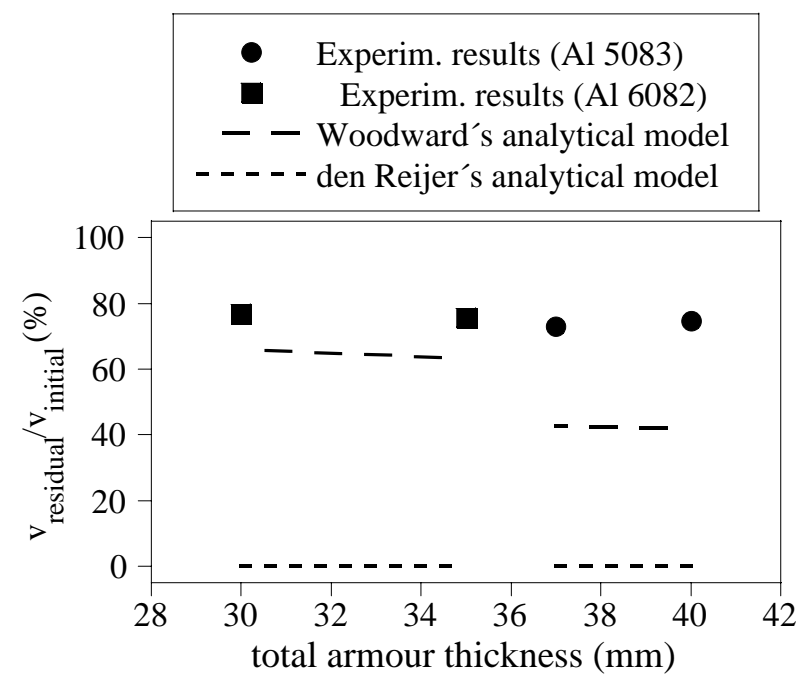

Figure 2. Analytical and experimental results of impact residual velocities of 20 APDS projectile $(\sim 50,000 \mathrm{~J})$ onto alumina $95 \%$ / aluminium alloy (5083 and 6082).

Three analytical models were developed (Florence in 1969 [1]; Woodward in 1990 [2]; den Reijer in 1991 [3]), but all of them have shortcomings that limit their usefulness as design tools. While most projectiles have conical or pointed heads, and impact on the armour at a more or less oblique angle, these models take account only of cylindrical projectiles and normal impact. In addition to this, the results are no longer valid for impact energies above 10,000 joules -medium caliber projectiles-, as shown in Fig. 2. And calibration is required to adjust the parameters defining the resistance behaviour of the ceramic material. In a recent symposium, Walker et al. [4] presented the first results obtained with a new model that is being developed. 
To cover these weaknesses, we have developed a new model that allows the simulation of both normal and oblique impacts of projectiles of small and medium caliber and of non-cylindrical geometries. Calibration has been greatly reduced since the model uses material properties readily available in the literature. With these developments, the simulation tool can now work out an impact problem in a few seconds. This article outlines the main aspects of the advance, the features of the model, the effects obtained, and the range of its usefulness.

\section{Development of the analytical model}

\subsection{Origin of the model}

All the hypotheses adopted were obtained from three sources: first, the earlier analytical models which provided the example for the simulation. The second source was the series of results of the ballistic tests carried out by the company “Empresa Nacional Santa Bárbara”. Many of these tests were made with ultra-rapid X-ray equipment, providing a sequence of $\mathrm{X}$-rays of each impact (Fig. 3).

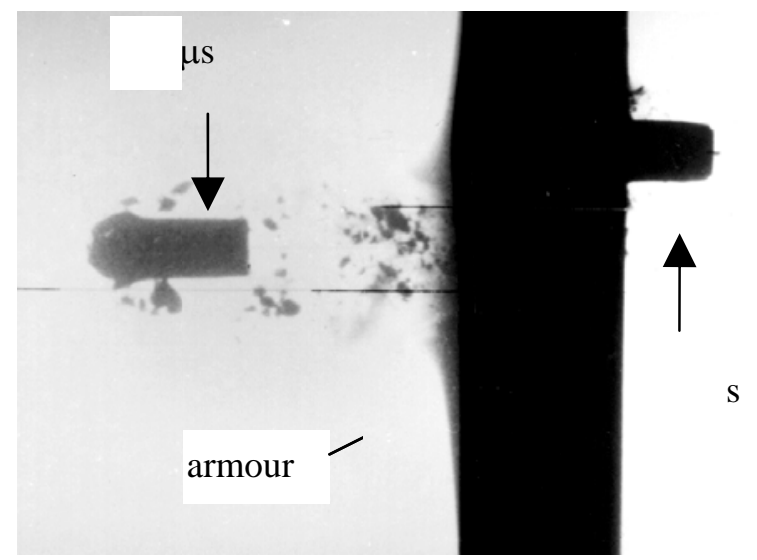

Figure 3. Two superimposed (and slightly displaced) images of the impact of a medium caliber projectile at 12 and $115 \mu$ s (Empresa Nacional Santa Bárbara).

From these images it is possible to determine the exact position and the velocity of the projectile and of the metal plate during penetration. In addition to these quantitative data, the images give a clearer perception of what happens in the 100 microseconds of impact. Finally, we have made simulations of some of these tests using a commercial finite difference hydrocode designed for impulse problems. The code was first checked with a set of independent test results, which gave detailed information on displacement, velocity and stress during penetration. This information was invaluable when it came to selecting the hypotheses to be adopted in the analytical model.

\subsection{Description of the model}

\subsubsection{Normal impact}

The penetration process can be divided into three phases (Fig. 4). The first is the stage of fragmentation of the ceramic material which occurs with the contact of the projectile on the armour. The stage of penetration of the ceramic material comes a few microseconds after contact; the ceramic material in front of the projectile starts to distribute the load on the metallic backplate which arrests the fragments, continues the erosion of the projectile and absorbs its kinetic energy. When the projectile has eliminated the ceramic cone, it meets the plate and initiates the stage of penetration of the metallic plate. If the energy of the projectile is high, it will perforate the panel; if not, it will be arrested at any of the three stages.

The special features of each of the three stages means that they must be modelled separately. A set of equations was formulated for each one, given that the interaction between the projectile and the target, the response of the ceramic material, and the behaviour of the metallic backing plate are completely different.

The choice of the hypotheses introduced into the model for each of the stages is the most critical part of the development. The approximation 
obtained with this type of model depends largely on its complexity. To introduce a large number of variables gives a more precise reproduction of the physical phenomena involved in the process, but this complicates the solution of the system of differential equations and means more time spent in calculation. So a balance has to be struck between the ability of the model to reproduce the experimental results and the need for a simple method of calculation that does not acquire the complexity of a finite element or a finite difference model.

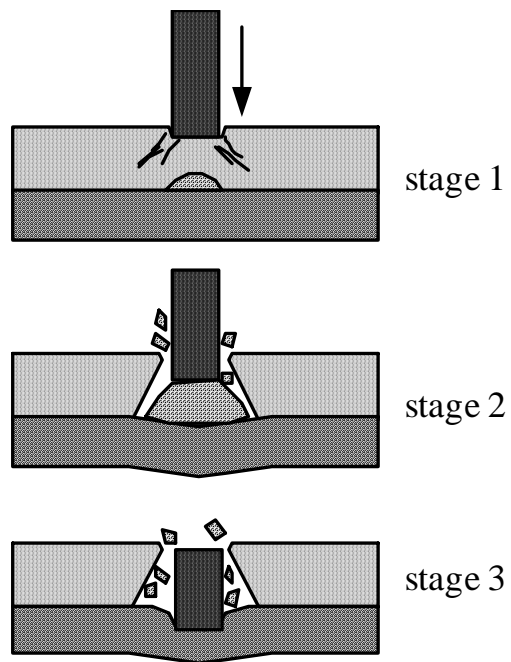

Figure 4. Stages of the penetration process.

To make the model useful as a preliminary design tool, care was taken to propose equations for material behaviour whose parameters are easily determined by experiment or are available in the literature. The other consideration was the shape of the projectile, since that of a cylinder with equivalent mass as in earlier models was seen to be an oversimplification for the projectiles used which have long conical heads.

The final result is a system of non-linear second order differential equations for each phase, to be integrated in time.

The equations of the model are derived from the application of different principles of physics to the three systems that enter into the problem: the projectile, the ceramic and the metal plate. For the first two systems, the equations are an application of the variation of linear momentum in the impact direction, bearing in mind that both these systems are of variable mass from the continuous erosion of both the projectile and the ceramic. For the metal plate, a balance is applied between the energy dissipated in its plastic deformation, the acquired kinetic energy and the work done by the ceramic cone. These equations are completed by the laws defining the mechanical properties of the materials.

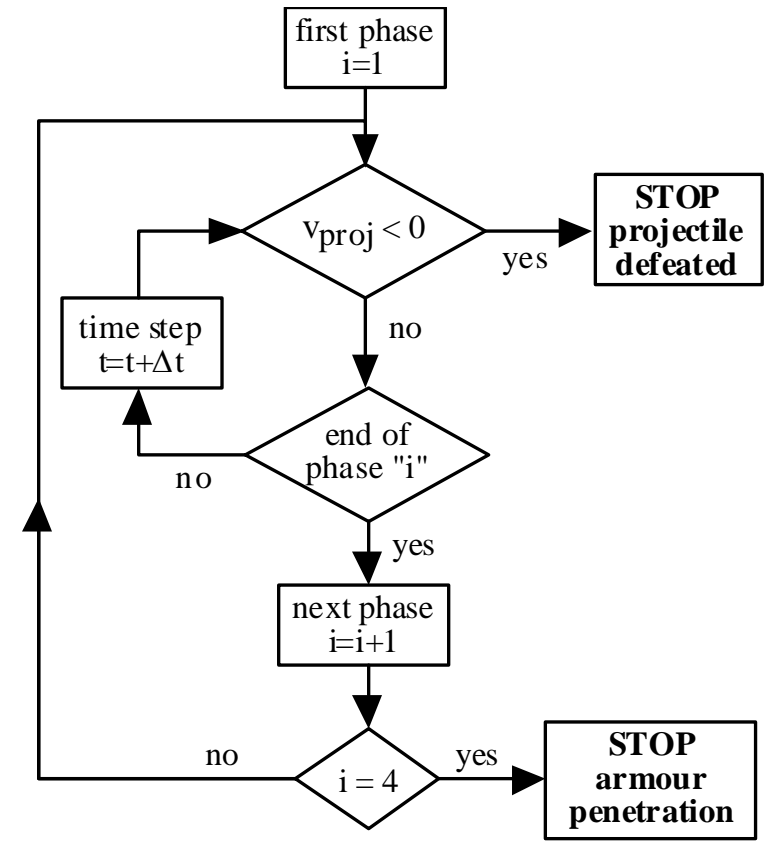

Figure 5. Diagram of calculation of a simulation of an impact problem. $v_{\text {proj }}$ represents the velocity of the projectile and " $\mathrm{i}$ " the number of the phase of the penetration process.

A Fortran code was programmed that allows a calculation at each step of the integration of the derivatives of the eight variables that define the problem from their values at an instant " $t$ ". Once these values are obtained, the code obtains the new values in an instant " $t+\Delta t$ " by the RungeKutta fourth order method. Together with this sub-routine, a modulus was programmed to control the process, verifying at each step of 
integration whether the stage should continue or stop to admit the following one with the new set of equations (Fig. 5).

A description of the penetration process, the equations of the model, and a detailed account of the physical aspects are given in the article by Zaera \& Sánchez-Galvez [5].

\subsubsection{Oblique impact}

The final contribution of the model is a rule that allows the analytical model to be used to calculate oblique impacts which in practice are much more frequent than normal ones. In the design of protections, panels are used, placed at an angle to the impact direction. So this new capacity is a useful addition to the analytical model.

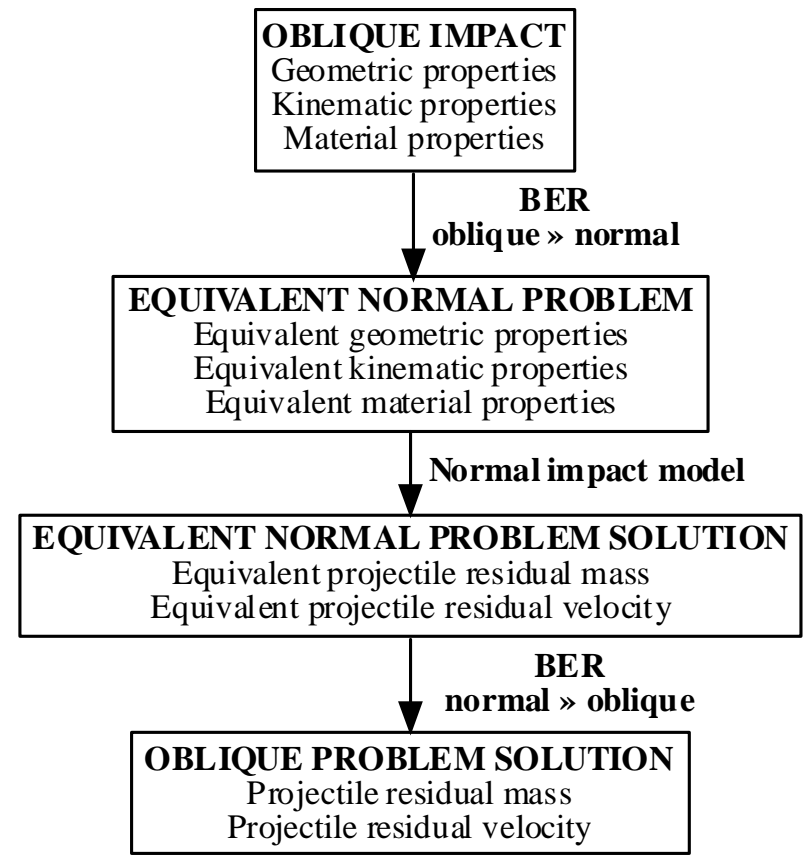

Figure 6. Diagram of a Ballistic Equivalence Rule.

Oblique impact is obviously a threedimensional phenomenon and there is no possibility of finding symmetries that would reduce it to a bidimensional one. Simulation by finite elements or finite differences is possible only if the code allows the generation of threedimensional meshes, and analytical simulation is equally complex. The usual solution is to transform the oblique impact into an equivalent normal one, applying a Ballistic Equivalence Rule (BER) as shown in Fig. 6. This transformation is made through a series of conversions. Once the new problem is determined, it is solved by the available analytical model for normal impact. The last step is to transform the solution by using another ballistic equivalence rule which gives the result of the original problem.

\section{Verification and validation of the model}

After a careful check of the implementation of the algorithms of control and of integration of the equations, of the input of data and the output of results, the verification of the analytical model was undertaken.

With slight variations of the input data, the model gave the conforming results. The correct evolution of the variables throughout the integration process was checked with the results obtained from the simulation by the code of finite differences. The precision afforded by this type of code, and the comprehensive information provided as regards displacement fields and velocity, means that it can be used as a fairly reliable and worthwhile reference. The evolution of the displacements and velocities with time that were obtained from both models were fairly close, except in the final stage of the impact process when the energy of the system is a good deal lower and errors are less important.

Then the validation was made of the predictive capacity of the model with a simulation by the analytical code of a number of test results different from those used in the stage of development, covering a wide range of projectiles, ceramic materials, aluminium alloys and impact angles. All showed errors in the residual kinetic energy of the projectile, below 
that demanded by the companies that had financed the project. Figs. 7 and 8 show the results of a series of impacts of a $20 \mathrm{~mm}$ APDS projectile, with a wolfram nucleus, 72 g mass, and initial firing speed of $1,200 \mathrm{~m} / \mathrm{s}$. The velocity and mass are both expressed divided by initial values.

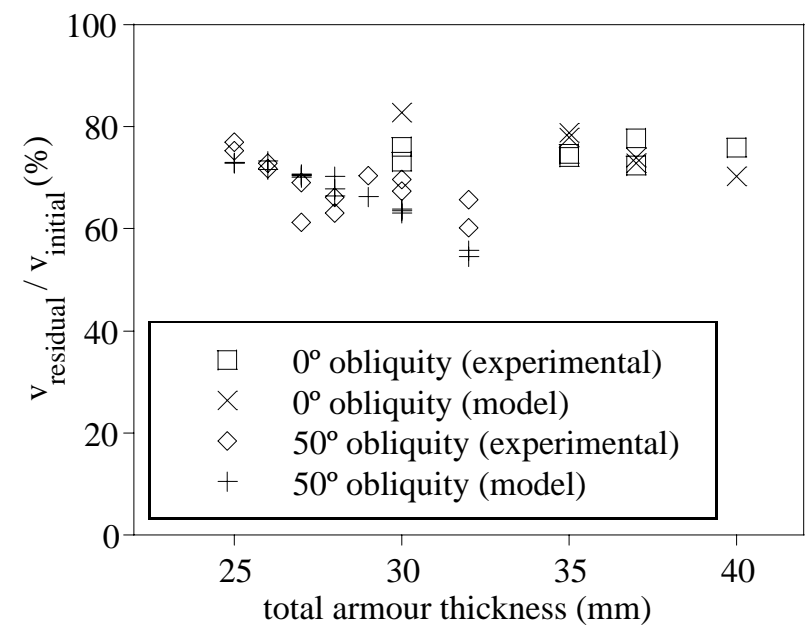

Figure 7. Analytical and experimental results of projectile residual velocities of 20 APDS projectile onto alumina 95\% / aluminium alloy.

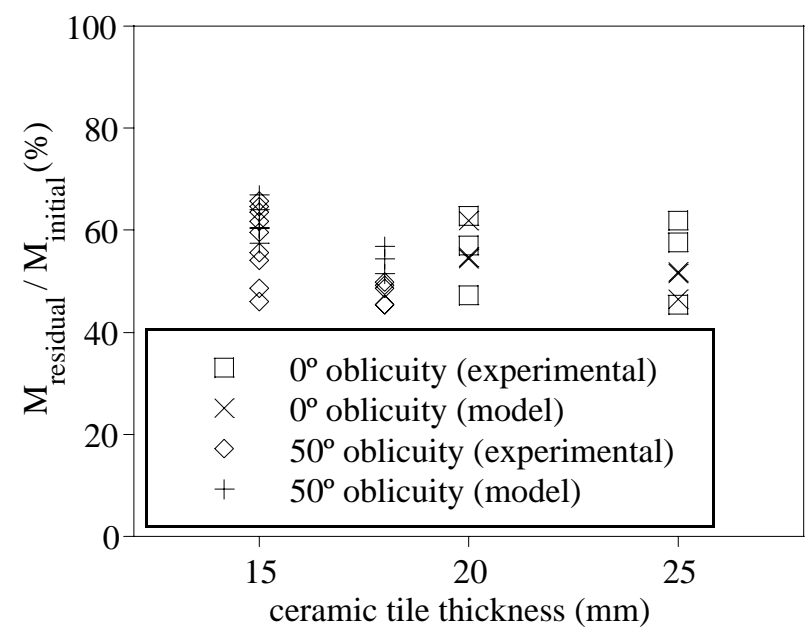

Figure 8. Analytical and experimental results of projectile residual masses of 20 APDS projectile onto alumina 95\% / aluminium alloy.

In addition to the experimental results obtained by the "Empresa Nacional Santa Bárbara", others were taken from scientific sources. All the data available allowed a check to be made of the model with different types of ceramic materials (aluminas of $85 \%, 95 \%, 98 \%$ and $99.5 \%$ purity, aluminium nitride) with different metals (aluminium alloy, steel), with projectiles of different calibers and materials (7.62 AP, 12.70 AP, 20 APDS, 25 APDS, 30 APDS), as well as different impact angles $\left(0^{\circ}, 50^{\circ}, 60^{\circ}, 65^{\circ}\right.$ NATO). The results are acceptable provided that:

i) The projectiles are not shattered on impact. A correct simulation of the results was not possible in the case of the 30 APDS because this projectile has a cylindrical hollow at the rear which causes its fragmentation during impact. The hypothesis of the model is unfounded once fragmentation occurs.

ii) The angle of impact is not very high. The Ballistic Equivalence Rule has given sufficiently exact results up to about $65^{\circ}$ between the impact direction and the normal to the armour surface - NATO criterion-. The possible ricochet at a greater angle of impact is not counted among the hypotheses of the model.

For medium caliber projectiles, the accuracy of the developed model is very superior to that of the earlier analytical models. These were already reliable enough for small caliber projectiles (type $7.62 \mathrm{AP}$ ), and the new model gives the same accuracy.

\section{Using the model as a preliminary design tool}

The nucleus of the calculation was completed with a graphic user interface programmed in Visual Basic which simplifies data input and the interpretation of the results of the simulation. The result is a tool for the preliminary design of ceramic/metal armours called SCARE (aSsessment of Ceramic ARmours Efficiency) which permits a simulation in a few seconds of 
calculation with a personal computer of whether the protection will arrest the projectile or be perforated, and in the latter case the velocity and residual mass of the projectile.

The problem for the engineer in designing the panel that is to protect the aircraft or armoured vehicle is sketched in Fig. 9. Knowing the type of projectile to be arrested, the firing distance, the impact obliquity and the reduction of the kinetic energy to be achieved, the design has to determine the materials and thicknesses of the panel that will give the required reduction of this energy. And it is at this stage where a simulation tool is called for to provide results in the shortest time as there are numerous combinations that can solve the problem.

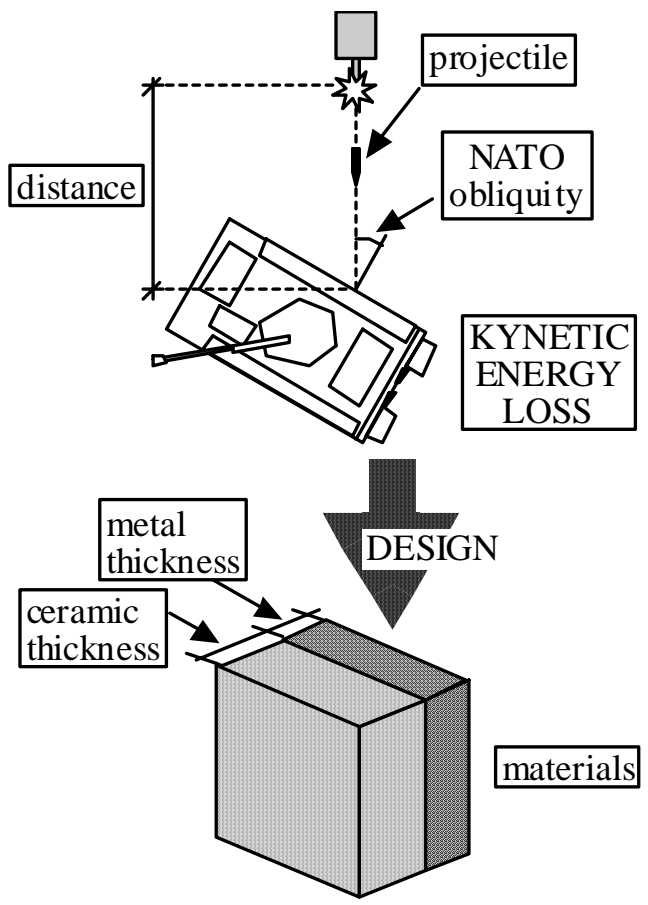

Figure 9. Factors affecting armour design.

With the SCARE code, graphics are obtained, of the types shown in Figs. 10 and 11. Knowing the type of projectile, the velocity and angle of impact, and the materials to be used in the manufacture of the armour, the graphics present the various combinations of ceramic/metal thicknesses that will produce the required loss of kinetic energy.

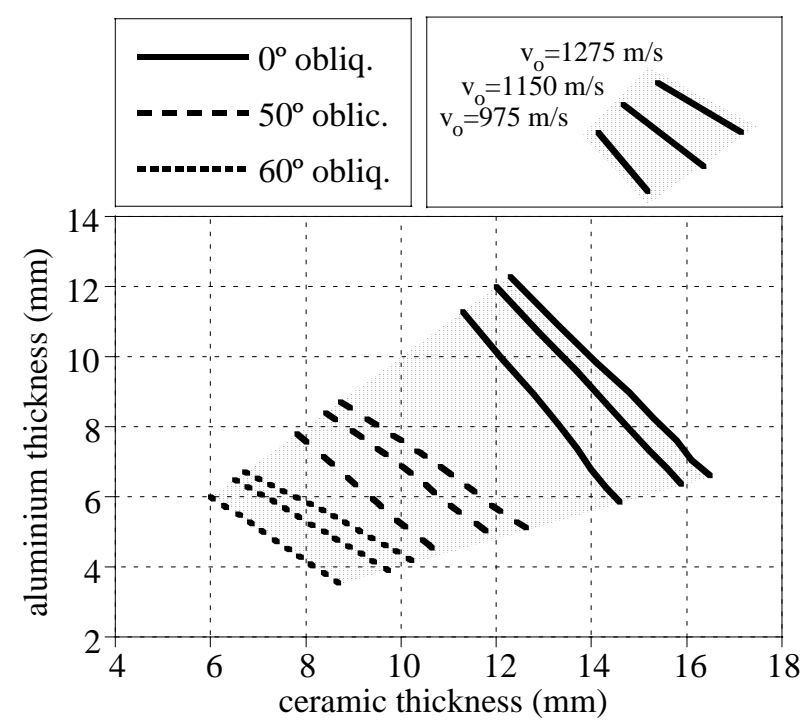

Figure 10. Design calculation of add-on protections. $25 \mathrm{~mm}$ APDS projectile; armour of aluminium nitride / 2017-T4 aluminium alloy; kinetic energy loss $40 \%$.

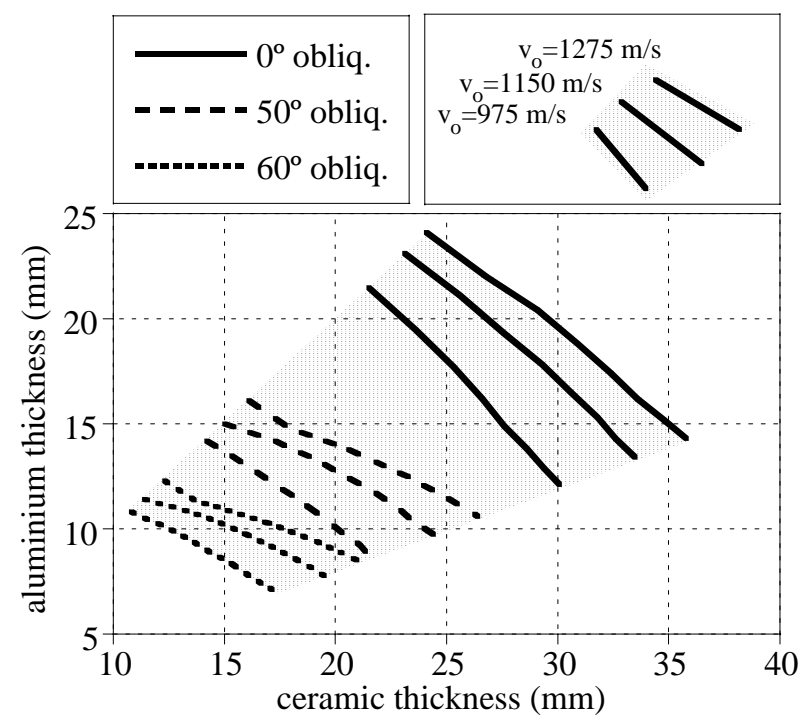

Figure 11. Design calculation of add-on protections. $20 \mathrm{~mm}$ APDS projectile; armour of aluminium nitride / 2017-T4 aluminium alloy; kinetic energy loss $100 \%$.

Where the complete arrest of the projectile is not required, it may be necessary to know also the residual velocity to design the main armour of the vehicle, behind the ceramic/metal panel. 
Then it may be useful to have graphics such as that shown in Fig. 12, showing the residual velocities of the projectile for different combinations of thickness of ceramic material and metal. The creation of this graphic (and the two previous ones) required about 15 minutes calculation with the model, using a Pentium computer.

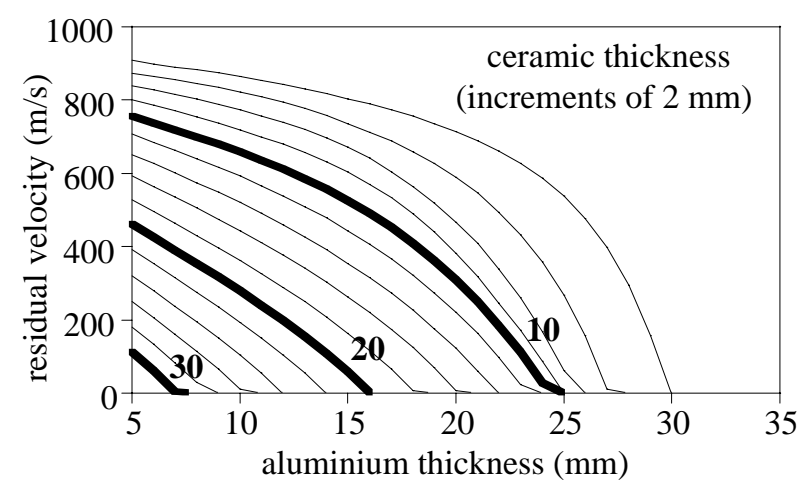

Figure 12. Design calculation of add-on protections. $14.5 \mathrm{~mm}$ AP projectile; impact velocity $975 \mathrm{~m} / \mathrm{s}$; armour of alumina / 2017-T4 aluminium alloy; normal impact.

Finally it is interesting to compare the results obtained from the analytical model with those from the finite differences code which is much more sophisticated.

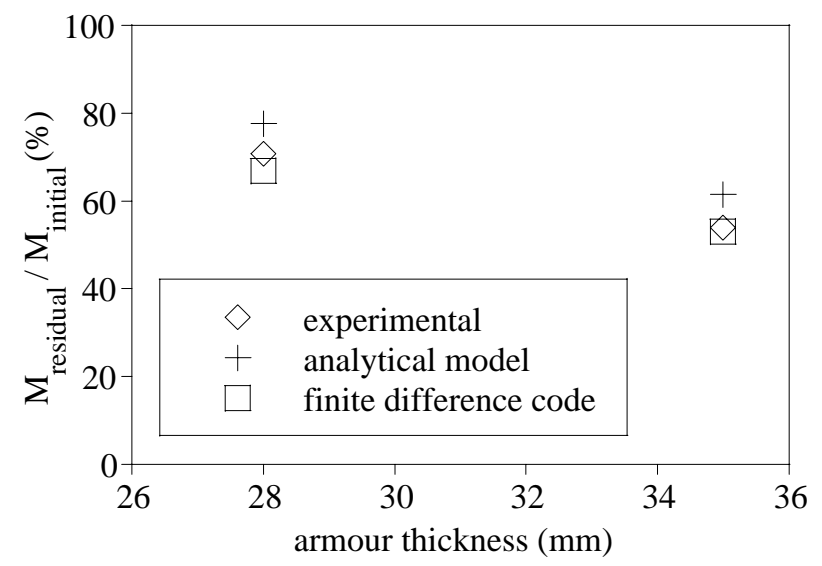

Figure 13. Simulated and experimental results of projectile residual masses of 20 APDS projectile onto alumina 95\% / aluminium alloy.

Fig. 13 shows the test results of residual masses of normal impact of a $20 \mathrm{~mm}$ APDS projectile on an alumina/aluminium armour. The finite difference code gives a slightly closer approximation than does the analytical model, but with the drawback that each of its simulations required 6-8 hours of calculation compared with the 2 seconds for the analytical model (both carried out with a Pentium).

\section{Conclusions}

A model has been developed for the simulation of normal or oblique impact of projectiles on light Ceramic/metal armours. It has been validated for different combinations of ceramic materials and metals as well as for projectiles of various materials, shapes and impact energies (from 2,000 to 100,000 J). The model has shown acceptable accuracy in predicting test results.

Given the speed with which the model simulates an impact problem, its usefulness is found in the initial stages of design: i.e. for the choice of materials and the determination of the thicknesses of the armour that will reduce the energy of the projectile. The complete design process will imply, however, real fire tests and simulations with more accurate codes -finite elements or finite differences-.

Since the model provides a simulation of the interaction between the projectile and the armour, it serves also to select the form and the material of the projectile most likely to improve its performance.

\section{Future Enhancements.}

This work is to be extended in two directions: that of the simulation model and that of the preliminary design tool.

To improve the model, attention will be given to the effect of multiple impacts on areas near the panel, to which ceramic armours are very sensitive. Since the ceramic/metal panels are 
normally added over the main armour of the vehicle, another aim is to develop a model of the penetration of a thick metal plate so that the simulation would include the main armourplating as well as the light-weight outer protection.

As regards the preliminary design tool, the aim is to extend its range by introducing subroutines to assist the choice of materials and the geometry of the armour in accordance with considerations of cost and of weight. The goal is a preliminary design tool with which to design the whole armour-plating of a vehicle on the basis of its shape, the level of protection required in each part, the most likely chance of impact and the most probable angle.

\section{Acknowledgements}

The authors are indebted to the Research \& Development Department of Empresa Nacional Santa Bárbara (Spain) for the performance of the ballistic tests and the X-ray shadowgraphs. This work is a part of the EUCLID RTP 3.2 project, supported by the Ministries of Defence of Spain, Denmark, Holland and Italy. The above project has been developed with the collaboration of several companies and research centres of the aforementioned countries.

\section{References}

[1] Florence A. L., "Interaction of projectiles and composite armour. Part II", Stanford Research Institute, Menlo Park, California, U.S.A., (1969).

[2] Woodward R. L.,"A simple one-dimensional approach to modelling ceramic composite armour defeat", Int. J. Impact Engng., (1990).

[3] den Reijer P. C., "Impact on Ceramic Faced Armours", PhD. Thesis, Delft University of Technology, (1991).

[4] Walker J. D., Anderson C. E. Jr., "An analytical model for ceramic-faced light armors", in Proceedings on the 16h International Symposium on Ballistics, San Francisco, (1996).
[5] Zaera R., Sánchez-Gálvez V. 1998. “Analytical modelling of normal and oblique ballistic impact on ceramic/metal lightweight armours”, Int. Journal Impact Engng, 21.

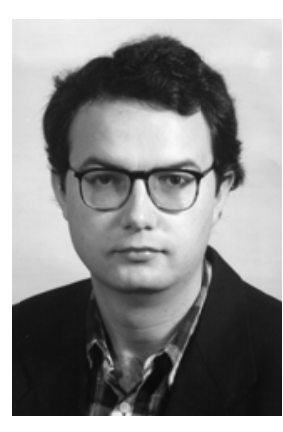

RAMÓN ZAERA POLO, lecturer in Structural Engineering at the Carlos III University of Madrid, graduated in Civil Engineering at the Polytechnic University of Madrid and obtained his doctorate of Civil Engineering in 1997. His current research interests lie in the areas of simulation of impact problems, dynamic characterization of materials and structural vibrations.

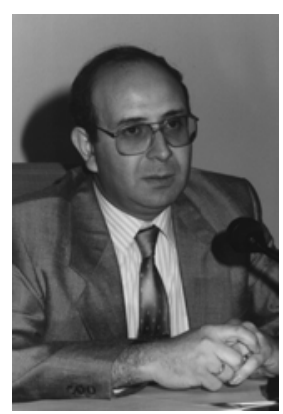

VICENTE SÁNCHEZ GÁLVEZ, Head of the Departament of Materials Science of the Polytechnic University of Madrid, graduated in Civil Engineering at this same university and in Physics at the Complutense, and obtained a doctorate in Civil Engineering in 1975. He has directed more than 20 public and private research projects in different areas of mechanical properties of materials as well as a number of doctoral theses. The author of numerous papers on this subject in international journals and of two books, he has edited the proceedings of three international symposia. He is consultant a permanent guest of the Ballistic Committee. 